\title{
SOLUTIONS OF QUASILINEAR WAVE EQUATION WITH STRONG AND NONLINEAR VISCOSITY
}

\author{
Jin-Soo Hwang, Shin-IChi Nakagiri, And Hiroki Tanabe
}

\begin{abstract}
We study a class of quasilinear wave equations with strong and nonlinear viscosity. By using the perturbation method for semilinear parabolic equations, we have established the fundamental results on existence, uniqueness and continuous dependence on data of weak solutions.
\end{abstract}

\section{Introduction}

Let $\Omega$ be an open bounded set of $\mathbb{R}^{n}$ with the smooth boundary $\Gamma$. We set $Q=(0, T) \times \Omega, \Sigma=(0, T) \times \Gamma$ for $T>0$. In our previous paper [7] we have studied the model equation of motion of membrane with strong viscosity described by

$$
\left\{\begin{array}{l}
\frac{\partial^{2} y}{\partial t^{2}}-\operatorname{div}\left(\frac{\nabla y}{\sqrt{1+|\nabla y|^{2}}}\right)-\mu \Delta \frac{\partial y}{\partial t}=f \quad \text { in } Q, \\
y=0 \text { on } \Sigma, \\
y(0, x)=y_{0}(x), \quad \frac{\partial y}{\partial t}(0, x)=y_{1}(x) \quad \text { in } \Omega,
\end{array}\right.
$$

where $\mu>0$ and $f$ is a forcing function. Based on the existence, uniqueness and continuity result of solutions for (1.1) in [7], we have solved the associated optimal control and identification problems in [8], [9]. As is well known, damping mechanism for the motion of membrane appears extensively and there are many factors of it. We classify the mechanism largely by air and structural factors, and the modified problem (1.1) is considered to be linear structurally damped case (cf. [1], [2], [12], etc.).

However, damping mechanism often occurs as nonlinear function of damping effect. In realistic sense, the Kirchhoff stress term $G(y)=\frac{\nabla y}{\sqrt{1+|\nabla y|^{2}}}$ may depend on position $x$ and time $t$. Thus we study a more general quasilinear

Received May 20, 2010.

2010 Mathematics Subject Classification. 35B30, 35L70.

Key words and phrases. equation of membrane with strong viscosity, weak solution, variational method. 
nonautonomous wave equation with structural and nonlinear dampings

$$
\begin{aligned}
& \frac{\partial^{2} y}{\partial t^{2}}-\operatorname{div}\left(G(t, x, \nabla y)+a(t, x) \nabla \frac{\partial y}{\partial t}\right) \\
= & F\left(t, x, y, \nabla y, \frac{\partial y}{\partial t}, \nabla \frac{\partial y}{\partial t}\right)+f,
\end{aligned}
$$

where $a(t, x)>0$ is a time dependent, space variable diffusion coefficient, $G(t, x, \nabla y)$ is a nonlinear function of principal vibration term and $F(t, x, y, \nabla y$, $\left.y^{\prime}, \nabla y^{\prime}\right)$ is a nonlinear damping term depending also on states.

As is recognized, the quasilinear equation like as (1.2) requires more manipulations in the analysis of systems as in [4], [5] and [10]. In this paper we propose an effective method to solve (1.2) by using the perturbation method for semilinear parabolic equations. The method strongly depends on the form of strong viscosity term $\operatorname{div}\left(a(t, x) \nabla \frac{\partial y}{\partial t}\right)$ and artificial technique to derive the energy estimates. Our aim of this paper is to establish the results on existence, uniqueness and continuity on data of weak solutions of (1.2) as in the framework of variational method in Dautray and Lions [2]. The proofs of results are quite different from those in [7] and give new proofs for the generalized quasilinear equation (1.2). The most important idea of the proofs is to derive the proper energy inequality for (1.2) by means of that for semilinear parabolic equations in [13] and the generalized Gronwall inequality. The existence proof is completed by using the strong convergence of successive approximations and the method of steps based on the time dependent energy inequality. Finally we note that the equation (1.2) covers the variational equation for (1.1) which will be used for the Gâteaux differentiability of solution mapping on data $y_{0}, y_{1}, f$ as in $[6]$.

\section{Preliminaries}

In this section we give preliminary results on parabolic problem and generalized Gronwall's inequality. We denote the scalar products and norms on Hilbert spaces $L^{2}(\Omega)$ and $H_{0}^{1}(\Omega)$ by $(\phi, \psi),|\phi|$ and $(\phi, \psi)_{H_{0}^{1}(\Omega)},\|\phi\|$, respectively. The scalar product and norm on $\left[L^{2}(\Omega)\right]^{n}$ are also denoted by $(\phi, \psi)$ and $|\phi|$. Then the scalar product $(\phi, \psi)_{H_{0}^{1}(\Omega)}$ and the norm $\|\phi\|$ of $H_{0}^{1}(\Omega)$ are given by $(\nabla \phi, \nabla \psi)$ and $\|\phi\|=|(\nabla \phi, \nabla \phi)|^{\frac{1}{2}}$, respectively. The duality pairing between $H_{0}^{1}(\Omega)$ and $H^{-1}(\Omega)$ is denoted by $\langle\phi, \psi\rangle$.

First, we consider the following parabolic problem

$$
\left\{\begin{array}{l}
\frac{\partial w}{\partial t}-\operatorname{div}(a(t, x) \nabla w)=\tilde{F}(t, x, w, \nabla w)+f \quad \text { in } Q, \\
w=0 \text { on } \Sigma, \\
w(0, x)=w_{0}(x) \text { in } \Omega,
\end{array}\right.
$$

where $w_{0} \in L^{2}(\Omega), f \in L^{2}\left(0, T ; H^{-1}(\Omega)\right)$, and $a(t, x)>0$ satisfies

$$
a(t, x) \geq \alpha>0, \quad \forall(t, x) \in Q, \quad a(\cdot) \in C\left([0, T] ; L^{\infty}(\Omega)\right) .
$$


In (2.1), the nonlinear function $\tilde{F}:[0, T] \times \Omega \times \mathbb{R} \times \mathbb{R}^{n} \rightarrow \mathbb{R}$ is assumed to satisfy the following conditions:

$(\tilde{F}-\mathrm{i}) \quad \tilde{F}(\cdot, \cdot, w, \xi)$ is measurable in $Q$ for each fixed $w \in \mathbb{R}$ and $\xi \in \mathbb{R}^{n}$,

$\left(\tilde{F}\right.$-ii) there is a $\tilde{\beta} \in L^{2}\left(0, T ; L^{\infty}(\Omega)\right)$ such that

$$
\begin{array}{r}
\left|\tilde{F}(t, x, w, \xi)-\tilde{F}\left(t, x, w^{\prime}, \xi^{\prime}\right)\right| \leq \tilde{\beta}(t, x)\left(\left|w-w^{\prime}\right|+\left|\xi-\xi^{\prime}\right|\right), \\
\text { a.e. }(t, x) \in Q, \forall w, w^{\prime} \in \mathbb{R}, \forall \xi, \xi^{\prime} \in \mathbb{R}^{n},
\end{array}
$$

$\left(\tilde{F}\right.$-iii) there is a $\tilde{\gamma} \in L^{2}\left(0, T ; L^{2}(\Omega)\right)$ such that

$$
|\tilde{F}(t, x, 0,0)| \leq \tilde{\gamma}(t, x), \quad \text { a.e. }(t, x) \in Q .
$$

Associated with $a(t, x)$, we define the bilinear form

$$
a(t ; \phi, \psi)=\int_{\Omega} a(t ; x) \nabla \phi(x) \nabla \psi(x) d x, \quad \forall \phi, \psi \in H_{0}^{1}(\Omega) .
$$

It is easily verified by (2.2) and (2.3) that

$$
a(t ; \phi, \phi) \geq \alpha\|\phi\|^{2} \text { for all } \phi \in H_{0}^{1}(\Omega) \text { and } t \in[0, T] \text {. }
$$

Then we can define the operator $A(t) \in \mathcal{L}\left(H_{0}^{1}(\Omega), H^{-1}(\Omega)\right)$ through the relation

$$
a(t ; \phi, \varphi)=\langle A(t) \phi, \varphi\rangle \text { for all } \phi, \varphi \in H_{0}^{1}(\Omega) .
$$

Further, for the above $\tilde{F}(t, x, w, \xi)$, we define the nonlinear function $\tilde{F}:[0, T] \times$ $H_{0}^{1}(\Omega) \rightarrow L^{2}(\Omega)$ (same symbol) by

$$
\tilde{F}(t, \phi)(x)=\tilde{F}(t, x, \phi(x), \nabla \phi(x)), \quad \text { a.e. } x \in \Omega, \quad \forall \phi \in H_{0}^{1}(\Omega) .
$$

Then from $(\tilde{F}$-ii) and ( $\tilde{F}$-iii), by applying Minkowski's inequality we have

$$
\begin{aligned}
& \left|\tilde{F}\left(t, w_{1}\right)-\tilde{F}\left(t, w_{2}\right)\right| \\
= & \left(\int_{\Omega}\left|\tilde{F}\left(t, x, w_{1}(x), \nabla w_{1}(x)\right)-\tilde{F}\left(t, x, w_{2}(x), \nabla w_{2}(x)\right)\right|^{2} d x\right)^{\frac{1}{2}} \\
\leq & \left(\int_{\Omega}\left(\tilde{\beta}(t, x)\left|w_{1}(x)-w_{2}(x)\right|+\tilde{\beta}(t, x)\left|\nabla w_{1}(x)-\nabla w_{2}(x)\right|\right)^{2} d x\right)^{\frac{1}{2}} \\
\leq & \left(\int_{\Omega} \tilde{\beta}(t, x)^{2}\left|w_{1}(x)-w_{2}(x)\right|^{2} d x\right)^{\frac{1}{2}} \\
& +\left(\int_{\Omega} \tilde{\beta}(t, x)^{2}\left|\nabla w_{1}(x)-\nabla w_{2}(x)\right|^{2} d x\right)^{\frac{1}{2}} \\
= & \tilde{\beta}(t)\left(\left|w_{1}-w_{2}\right|+\left\|w_{1}-w_{2}\right\|\right) \leq\left(c_{1}+1\right) \tilde{\beta}(t)\left\|w_{1}-w_{2}\right\|
\end{aligned}
$$

and

$$
|\tilde{F}(t, 0)| \leq \tilde{\gamma}(t), \quad \text { a.e. } t \in[0, T]
$$

where $\tilde{\beta}(t)=\|\tilde{\beta}(t, \cdot)\|_{L^{\infty}(\Omega)}, \tilde{\gamma}(t)=\|\tilde{\gamma}(t, \cdot)\|_{L^{2}(\Omega)}$, and $c_{1}$ is the imbedding constant of $H_{0}^{1}(\Omega) \hookrightarrow L^{2}(\Omega)$. Thus, the evolution equation form of $(2.1)$ is 
given by

$$
\left\{\begin{array}{l}
w^{\prime}(t)+A(t) w(t)=\tilde{F}(t, w)+f(t), \quad 0 \leq t \leq T \\
w(0)=w_{0} \in L^{2}(\Omega) .
\end{array}\right.
$$

Definition 2.1. A function $w$ is said to be a weak solution of (2.1) (or (2.8)) if

$$
w \in L^{2}\left(0, T ; H_{0}^{1}(\Omega)\right) \cap C\left([0, T] ; L^{2}(\Omega)\right), w^{\prime} \in L^{2}\left(0, T ; H^{-1}(\Omega)\right)
$$

and $w$ satisfies

$$
\left\{\begin{array}{c}
\left\langle w^{\prime}(\cdot), \phi\right\rangle+a(\cdot ; \nabla w(\cdot), \nabla \phi)=(\tilde{F}(\cdot, w(\cdot)), \phi)+\langle f(\cdot), \phi\rangle \\
\quad \text { for all } \phi \in H_{0}^{1}(\Omega) \text { in the sense of } \mathcal{D}^{\prime}(0, T), \\
w(0)=w_{0} \in L^{2}(\Omega) .
\end{array}\right.
$$

The following proposition is proved in Wang and Nakagiri [13] under the conditions (2.6) and (2.7) on $\tilde{F}$ according to the argument in Dautray and Lions $[2]$.

Proposition 2.1. Assume that $w_{0} \in L^{2}(\Omega), f \in L^{2}\left(0, T ; H^{-1}(\Omega)\right)$ and $\tilde{F}$ satisfies $(\tilde{F}-\mathrm{i})-(\tilde{F}$-iii). Then the problem $(2.1)$ has a unique weak solution $w$ satisfying the following energy equality

$$
\begin{aligned}
& \frac{1}{2}|w(t)|^{2}+\int_{0}^{t} a(s ; w(s), w(s)) d s \\
= & \frac{1}{2}\left|w_{0}\right|^{2}+\int_{0}^{t}(\tilde{F}(s, w(s)), w(s)) d s+\int_{0}^{t}\langle f(s), w(s)\rangle d s, \quad \forall t \in[0, T] .
\end{aligned}
$$

Next, we prepare the following proposition on the generalized Gronwall's inequality (cf. [2, p. 559]).

Proposition 2.2. Let $\varphi$ be a function in $L^{\infty}(0, T), \varphi(t) \geq 0$, a.e. $t \in[0, T]$, $\mu$ be a function in $L^{1}(0, T), \mu(t) \geq 0$, a.e. $t \in[0, T]$, and $\delta$ be a function in $L^{\infty}(0, T), \delta(t) \geq 0$, a.e. $t \in[0, T]$. Assume

$$
\varphi(t) \leq \int_{0}^{t} \mu(\sigma) \varphi(\sigma) d \sigma+\delta(t), \quad \text { a.e. } t \in[0, T] .
$$

Then

$$
\varphi(t) \leq \delta(t)+\int_{0}^{t} \mu(s) \delta(s) \exp \left(\int_{s}^{t} \mu(\sigma) d \sigma\right) d s, \quad \text { a.e. } t \in[0, T]
$$




\section{Main results}

We study the following Dirichlet boundary value problem for the quasilinear wave equation with strong and nonlinear damping

$$
\left\{\begin{array}{l}
\quad \frac{\partial^{2} y}{\partial t^{2}}-\operatorname{div}\left(G(t, x, \nabla y)+a(t, x) \nabla \frac{\partial y}{\partial t}\right) \\
=F\left(t, x, y, \nabla y, \frac{\partial y}{\partial t}, \nabla \frac{\partial y}{\partial t}\right)+f \quad \text { in } Q \\
y=0 \quad \text { on } \Sigma, \\
y(0, x)=y_{0}(x), \quad \frac{\partial y}{\partial t}(0, x)=y_{1}(x) \quad \text { in } \Omega
\end{array}\right.
$$

where $f$ is a forcing function, $y_{0}$ and $y_{1}$ are initial data. In (3.1), we suppose that $a(t, x)$ satisfies $(2.2), f \in L^{2}\left(0, T ; H^{-1}(\Omega)\right), y_{0} \in H_{0}^{1}(\Omega)$ and $y_{1} \in L^{2}(\Omega)$. On the nonlinear term $G(t, x, \nabla y)$ in $(3.1)$, we assume that the function $G$ : $[0, T] \times \Omega \times \mathbb{R}^{n} \rightarrow \mathbb{R}^{n}$ satisfies the following conditions:

(G-i) $\quad G(\cdot, \cdot, z)$ is measurable in $Q$ for each fixed $z \in \mathbb{R}^{n}$,

(G-ii) $G(t, x, 0)=0$, a.e. $(t, x) \in Q$, and there is a $G_{0} \in L^{\infty}\left(0, T ; L^{\infty}(\Omega)\right)$ such that

$$
\begin{array}{r}
\left|G(t, x, z)-G\left(t, x, z^{\prime}\right)\right| \leq G_{0}(t, x)\left|z-z^{\prime}\right| \\
\text { a.e. }(t, x) \in Q, \forall z, z^{\prime} \in \mathbb{R}^{n} .
\end{array}
$$

For the above $G$, we define the nonlinear function $G(t, \nabla \cdot):[0, T] \times H_{0}^{1}(\Omega) \rightarrow$ $\left[L^{2}(\Omega)\right]^{n}$ by

$$
G(t, \nabla \phi)=G(t, x, \nabla \phi(x)) \text {, a.e. } x \in \Omega, \quad \forall \phi \in H_{0}^{1}(\Omega) .
$$

Finally, on the nonlinear term $F\left(t, x, y, \nabla y, \frac{\partial y}{\partial t}, \nabla \frac{\partial y}{\partial t}\right)$ in (3.1), we suppose that the function $F:[0, T] \times \Omega \times \mathbb{R} \times \mathbb{R}^{n} \times \mathbb{R} \times \mathbb{R}^{n} \rightarrow \mathbb{R}$ satisfying the following conditions:

(F-i) $\quad F(\cdot, \cdot, y, z, w, \xi)$ is measurable in $Q$ for each fixed $y, w \in \mathbb{R}$ and $z, \xi \in \mathbb{R}^{n}$,

(F-ii) there are $\beta_{1} \in L^{2}\left(0, T ; L^{\infty}(\Omega)\right)$ and $\beta_{2} \in L^{\infty}\left(0, T ; L^{\infty}(\Omega)\right)$ such that

$$
\begin{aligned}
&\left|F(t, x, y, z, w, \xi)-F\left(t, x, y^{\prime}, z^{\prime}, w^{\prime}, \xi^{\prime}\right)\right| \\
& \leq \beta_{1}(t, x)\left(\left|y-y^{\prime}\right|+\left|z-z^{\prime}\right|\right)+\beta_{2}(t, x)\left(\left|w-w^{\prime}\right|+\left|\xi-\xi^{\prime}\right|\right), \\
& \text { a.e. }(t, x) \in Q, \quad \forall y, y^{\prime}, w, w^{\prime} \in \mathbb{R}, \quad \forall z, z^{\prime}, \xi, \xi^{\prime} \in \mathbb{R}^{n},
\end{aligned}
$$

(F-iii) there is a $\gamma \in L^{\infty}\left(0, T ; L^{\infty}(\Omega)\right)$ such that

$$
\begin{aligned}
& |F(t, x, y, z, 0,0)| \leq \gamma(t, x)(|y|+|z|), \\
& \text { a.e. }(t, x) \in Q, \forall y \in \mathbb{R}, \forall z \in \mathbb{R}^{n} .
\end{aligned}
$$


For the above $F$, we can define the nonlinear map $F:[0, T] \times H_{0}^{1}(\Omega) \times H_{0}^{1}(\Omega) \rightarrow$ $L^{2}(\Omega)$ by

$$
F(t, y, w)(x)=F(t, x, y(x), \nabla y(x), w(x), \nabla w(x)), \text { a.e. } x \in \Omega
$$

for each $(y, w) \in H_{0}^{1}(\Omega) \times H_{0}^{1}(\Omega)$. Repeating similar calculations for (2.6) and (2.7), by (F-ii) and (F-iii) we can verify by using Minkowski's inequality the following estimates: For each $y_{1}, y_{2} \in H_{0}^{1}(\Omega)$ and $w_{1}, w_{2} \in H_{0}^{1}(\Omega)$,

$$
\begin{aligned}
& \left|F\left(t, y_{1}, w_{1}\right)-F\left(t, y_{2}, w_{2}\right)\right| \\
\leq & \left(c_{1}+1\right)\left(\beta_{1}(t)\left|\nabla y_{1}-\nabla y_{2}\right|+\beta_{2}(t)\left|\nabla w_{1}-\nabla w_{2}\right|\right),
\end{aligned}
$$

and for each $y \in H_{0}^{1}(\Omega)$,

$$
|F(t, y, 0)| \leq\left(c_{1}+1\right) \gamma(t)|\nabla y|, \quad \text { a.e. } t \in[0, T],
$$

where $\beta_{1}(t)=\left\|\beta_{1}(t, \cdot)\right\|_{L^{\infty}(\Omega)}, \beta_{2}(t)=\left\|\beta_{2}(t, \cdot)\right\|_{L^{\infty}(\Omega)}$ and $\gamma(t)=\|\gamma(t, \cdot)\|_{L^{\infty}(\Omega)}$. The solution space $W(0, T)$ of $(3.1)$ is defined by

$$
\left\{g \mid g \in L^{2}\left(0, T ; H_{0}^{1}(\Omega)\right), g^{\prime} \in L^{2}\left(0, T ; H_{0}^{1}(\Omega)\right), g^{\prime \prime} \in L^{2}\left(0, T ; H^{-1}(\Omega)\right)\right\}
$$

endowed with the norm

$$
\|g\|_{W(0, T)}=\left(\|g\|_{L^{2}\left(0, T ; H_{0}^{1}(\Omega)\right)}^{2}+\left\|g^{\prime}\right\|_{L^{2}\left(0, T ; H_{0}^{1}(\Omega)\right)}^{2}+\left\|g^{\prime \prime}\right\|_{L^{2}\left(0, T ; H^{-1}(\Omega)\right)}^{2}\right)^{\frac{1}{2}}
$$

(cf. Dautray and Lions [2, p. 471]). We remark that $W(0, T)$ is continuously imbedded in $C\left([0, T] ; H_{0}^{1}(\Omega)\right) \cap C^{1}\left([0, T] ; L^{2}(\Omega)\right)$ (cf. [2, p. 555]). The evolution equation form of (3.1) is described by

$$
\left\{\begin{array}{l}
y^{\prime \prime}(t)-\operatorname{div}\left(G(t, \nabla y(t))+A(t) y^{\prime}(t)=F\left(t, y(t), y^{\prime}(t)\right)+f(t), 0 \leq t \leq T\right. \\
y(0)=y_{0}, \quad y^{\prime}(0)=y_{1}
\end{array}\right.
$$

Definition 3.1. A function $y$ is said to be a weak solution of (3.1) (or (3.6)) if $y \in W(0, T)$ and $y$ satisfies

$$
\left\{\begin{array}{l}
\left\langle y^{\prime \prime}(\cdot), \phi\right\rangle+(G(\cdot, \nabla y(\cdot)), \nabla \phi)+a\left(\cdot ; \nabla y^{\prime}(\cdot), \nabla \phi\right) \\
=\left(F\left(\cdot, y(\cdot), y^{\prime}(\cdot)\right), \phi\right)+\langle f(\cdot), \phi\rangle \\
\text { for all } \phi \in H_{0}^{1}(\Omega) \text { in the sense of } \mathcal{D}^{\prime}(0, T) \\
y(0)=y_{0} \in H_{0}^{1}(\Omega), \quad y^{\prime}(0)=y_{1} \in L^{2}(\Omega)
\end{array}\right.
$$

The following theorems give the basic results on existence, uniqueness, regularity and continuity on data of the weak solution of (3.1), which extend those in Hwang and Nakagiri [7].

Theorem 3.1. Assume that $G$ satisfies (G-i)-(G-ii), F satisfies (F-i)-(F-iii), $f \in L^{2}\left(0, T ; H^{-1}(\Omega)\right)$ and $y_{0} \in H_{0}^{1}(\Omega), y_{1} \in L^{2}(\Omega)$. Then the problem (3.1) has a unique weak solution $y$ in $W(0, T)$. Furthermore, $y$ has the following estimate

$$
\left|y^{\prime}(t)\right|^{2}+|\nabla y(t)|^{2}+\int_{0}^{t}\left|\nabla y^{\prime}(s)\right|^{2} d s
$$




$$
\leq C\left(\left\|y_{0}\right\|^{2}+\left|y_{1}\right|^{2}+\|f\|_{L^{2}\left(0, T ; H^{-1}(\Omega)\right)}^{2}\right), \quad \forall t \in[0, T],
$$

where $C$ is a constant depending only on $\alpha>0, G_{0}, \beta_{1}, \beta_{2}, \gamma$ and $T>0$.

Let $P$ be a product space defined by

$$
P=H_{0}^{1}(\Omega) \times L^{2}(\Omega) \times L^{2}\left(0, T ; H^{-1}(\Omega)\right) .
$$

For each $p=\left(y_{0}, y_{1}, f\right) \in P$ we have a unique weak solution $y=y(p) \in$ $W(0, T)$ of $(3.1)$ by Theorem 3.1. Hence we can define the solution mapping $p=\left(y_{0}, y_{1}, f\right) \rightarrow y(p)$ of $P$ into $W(0, T)$.

Theorem 3.2. The solution mapping $p=\left(y_{0}, y_{1}, f\right) \rightarrow y(p)$ of $P$ into $W(0, T)$ is strongly continuous. Further, for each $p_{1}=\left(y_{0}^{1}, y_{1}^{1}, f_{1}\right) \in P$ and $p_{2}=$ $\left(y_{0}^{2}, y_{1}^{2}, f_{2}\right) \in P$ we have the inequality

$$
\begin{aligned}
& \left|y^{\prime}\left(p_{1} ; t\right)-y^{\prime}\left(p_{2} ; t\right)\right|^{2}+\left|\nabla y\left(p_{1} ; t\right)-\nabla y\left(p_{2} ; t\right)\right|^{2} \\
& +\int_{0}^{t}\left|\nabla y^{\prime}\left(p_{1} ; s\right)-\nabla y^{\prime}\left(p_{2} ; s\right)\right|^{2} d s \\
\leq & C\left(\left\|y_{0}^{1}-y_{0}^{2}\right\|^{2}+\left|y_{1}^{1}-y_{1}^{2}\right|^{2}+\left\|f_{1}-f_{2}\right\|_{L^{2}\left(0, T ; H^{-1}(\Omega)\right)}^{2}\right), \forall t \in[0, T] .
\end{aligned}
$$

\section{Proofs of main results}

We shall prove the main results by the perturbation method using the parabolic problem (2.1). To this end, we give the following observation. Let $w(t)$ be a unique weak solution of (2.1) with $w_{0}=y_{1} \in L^{2}(\Omega)$, and let

$$
y(t)=y_{0}+\int_{0}^{t} w(s) d s .
$$

Then $y^{\prime}=w, y^{\prime \prime}=w^{\prime}, y(0)=y_{0} \in H_{0}^{1}(\Omega), y^{\prime}(0)=y_{1} \in L^{2}(\Omega)$, and $y \in$ $W(0, T) \cap C^{1}\left([0, T] ; L^{2}(\Omega)\right)$. It is easily verified by Proposition 2.1 that $y$ is a unique weak solution of

$$
\left\{\begin{array}{l}
\frac{\partial^{2} y}{\partial t^{2}}-\operatorname{div}\left(a(t, x) \nabla \frac{\partial y}{\partial t}\right)=\tilde{F}\left(t, x, \frac{\partial y}{\partial t}, \nabla \frac{\partial y}{\partial t}\right)+f \quad \text { in } Q, \\
y=0 \quad \text { on } \Sigma, \\
y(0, x)=y_{0}(x), \quad \frac{\partial y}{\partial t}(0, x)=y_{1}(x) \quad \text { in } \Omega,
\end{array}\right.
$$

which satisfies the energy equality

$$
\begin{aligned}
& \frac{1}{2}\left|y^{\prime}(t)\right|^{2}+\int_{0}^{t} a\left(s ; y^{\prime}(s), y^{\prime}(s)\right) d s \\
= & \frac{1}{2}\left|y_{1}\right|^{2}+\int_{0}^{t}\left(\tilde{F}\left(s, y^{\prime}(s)\right), y^{\prime}(s)\right) d s+\int_{0}^{t}\left\langle f(s), y^{\prime}(s)\right\rangle d s, \quad \forall t \in[0, T] .
\end{aligned}
$$


Proof of Theorem 3.1. Let $\phi \in L^{2}\left(0, T ; H_{0}^{1}(\Omega)\right)$ be given. Then by (G-ii), we see

$$
\begin{aligned}
& |G(t, \nabla \phi(t))|^{2} \\
= & \int_{\Omega}|G(t, x, \nabla \phi(t, x))|^{2} d x \\
\leq & \left\|G_{0}(t, \cdot)\right\|_{L^{\infty}(\Omega)}^{2} \int_{\Omega}|\nabla \phi(t, x)|^{2} d x \leq\left\|G_{0}\right\|^{2}|\nabla \phi(t)|^{2}, \quad \text { a.e. } t \in[0, T],
\end{aligned}
$$

so that

$$
\nabla \phi \in L^{2}\left(0, T ;\left[L^{2}(\Omega)\right]^{n}\right), \quad G(\cdot, \nabla \phi) \in L^{2}\left(0, T ;\left[L^{2}(\Omega)\right]^{n}\right),
$$

where $\left\|G_{0}\right\|=\left\|G_{0}\right\|_{L^{\infty}\left(0, T ; L^{\infty}(\Omega)\right)}$. This implies

$$
\operatorname{div} G(\cdot, \nabla \phi) \in L^{2}\left(0, T ; H^{-1}(\Omega)\right) \text {. }
$$

Also, by (G-ii) for any $\phi_{1}, \phi_{2} \in L^{2}\left(0, T ; H_{0}^{1}(\Omega)\right)$ we have

$$
\left|G\left(t, \nabla \phi_{1}(t)\right)-G\left(t, \nabla \phi_{2}(t)\right)\right|^{2} \leq\left\|G_{0}\right\|^{2}\left|\nabla \phi_{1}(t)-\nabla \phi_{2}(t)\right|^{2}, \quad \text { a.e. } t \in[0, T] .
$$

For each $\phi \in L^{2}\left(0, T ; H_{0}^{1}(\Omega)\right)$, it is verified that the function $\tilde{F}=\tilde{F}_{\phi}$ defined by

$$
\tilde{F}_{\phi}:=F(t, x, \phi(t, x), \nabla \phi(t, x), w, \xi):[0, T] \times \Omega \times \mathbb{R} \times \mathbb{R}^{n} \rightarrow \mathbb{R}
$$

satisfies the conditions $(\tilde{F}-\mathrm{i})-(\tilde{F}$-iii) with

$$
\tilde{\beta}(t, x)=\beta_{2}(t, x) \in L^{\infty}\left(0, T ; L^{\infty}(\Omega)\right) \subset L^{2}\left(0, T ; L^{\infty}(\Omega)\right)
$$

and

$$
\tilde{\gamma}(t, x)=\gamma(t, x)(|\phi(t, x)|+|\nabla \phi(t, x)|) \in L^{2}\left(0, T ; L^{2}(\Omega)\right)
$$

by $\gamma \in L^{\infty}\left(0, T ; L^{\infty}(\Omega)\right)$ and $\phi,|\nabla \phi| \in L^{2}\left(0, T ; L^{2}(\Omega)\right)$. Hence, according to the former observation and (4.4) there exists a weak solution $z$ of the problem

$$
\left\{\begin{array}{l}
\quad \frac{\partial^{2} z}{\partial t^{2}}-\operatorname{div}\left(a(t, x) \nabla \frac{\partial z}{\partial t}\right) \\
=F\left(t, x, \phi, \nabla \phi, \frac{\partial z}{\partial t}, \nabla \frac{\partial z}{\partial t}\right)+\operatorname{div} G(t, x, \nabla \phi)+f \quad \text { in } Q \\
z=0 \quad \text { on } \Sigma, \\
z(0, x)=y_{0}(x), \quad \frac{\partial z}{\partial t}(0, x)=y_{1}(x) \quad \text { in } \Omega
\end{array}\right.
$$

satisfying $z \in W(0, T) \cap C^{1}\left([0, T] ; L^{2}(\Omega)\right)$, and such a solution is unique. The problem (4.7) can be written by the following evolution equation form

$$
\left\{\begin{array}{l}
z^{\prime \prime}(t)+A(t) z^{\prime}(t)=F\left(t, \phi(t), z^{\prime}(t)\right)+\operatorname{div}(G(t, \nabla \phi(t)))+f(t), 0 \leq t \leq T \\
z(0)=y_{0}, \quad z^{\prime}(0)=y_{1}
\end{array}\right.
$$

We seek for a fixed point of the mapping $\phi \mapsto z$ considered as a mapping from the set

$$
\mathcal{S}=\left\{\phi \in W^{1,2}\left(0, T ; H_{0}^{1}(\Omega)\right), \phi(0)=y_{0}\right\}
$$


to itself, where $z$ is the weak solution of (4.7) or (4.8). Let $u_{i} \in \mathcal{S}, i=1,2$ be given, and $v_{i}, i=1,2$, be the weak solution of

(4.9) $\left\{\begin{array}{l}v_{i}^{\prime \prime}(t)+A(t) v_{i}^{\prime}(t)=F\left(t, u_{i}(t), v_{i}^{\prime}(t)\right)+\operatorname{div}\left(G\left(t, \nabla u_{i}(t)\right)\right)+f(t), 0 \leq t \leq T \\ v_{i}(0)=y_{0}, \quad v_{i}^{\prime}(0)=y_{1} .\end{array}\right.$

Set

$$
u=u_{1}-u_{2}, \quad v=v_{1}-v_{2} .
$$

Then we see easily that

$$
u \in W^{1,2}\left(0, T ; H_{0}^{1}(\Omega)\right), \quad u(0)=0,
$$

and $v \in W(0, T) \cap C^{1}\left(0, T ; L^{2}(\Omega)\right)$ satisfies

$$
\left\{\begin{aligned}
v^{\prime \prime}(t)+A(t) v^{\prime}(t)= & F\left(t, u_{1}(t), v_{1}^{\prime}(t)\right)-F\left(t, u_{2}(t), v_{2}^{\prime}(t)\right) \\
& +\operatorname{div}\left(G\left(t, \nabla u_{1}(t)\right)-G\left(t, \nabla u_{2}(t)\right)\right), \quad 0 \leq t \leq T \\
v(0)=0, \quad v^{\prime}(0)=0 . &
\end{aligned}\right.
$$

Application of the energy equality (4.3) to $v$ and integration by parts yield

$$
\begin{aligned}
& \frac{1}{2}\left|v^{\prime}(t)\right|^{2}+\int_{0}^{t} a\left(s ; \nabla v^{\prime}(s), \nabla v^{\prime}(s)\right) d s \\
& -\int_{0}^{t}\left(F\left(s, u_{1}(s), v_{1}^{\prime}(s)\right)-F\left(s, u_{2}(s), v_{2}^{\prime}(s)\right), v^{\prime}(s)\right) d s \\
= & -\int_{0}^{t}\left(G\left(s, \nabla u_{1}(s)\right)-G\left(s, \nabla u_{2}(s)\right), \nabla v^{\prime}(s)\right) d s .
\end{aligned}
$$

By Schwarz inequality, we have

$$
\begin{aligned}
& \left|\int_{0}^{t}\left(G\left(s, \nabla u_{1}(s)\right)-G\left(s, \nabla u_{2}(s)\right), \nabla v^{\prime}(s)\right) d s\right| \\
\leq & \frac{1}{\alpha} \int_{0}^{t}\left|G\left(s, \nabla u_{1}(s)\right)-G\left(s, \nabla u_{2}(s)\right)\right|^{2} d s+\frac{\alpha}{4} \int_{0}^{t}\left|\nabla v^{\prime}(s)\right|^{2} d s .
\end{aligned}
$$

Further, by (4.5) we deduce

$$
\begin{gathered}
\int_{0}^{t}\left|G\left(s, \nabla u_{1}(s)\right)-G\left(s, \nabla u_{2}(s)\right)\right|^{2} d s \leq\left\|G_{0}\right\|^{2} \int_{0}^{t}|\nabla u(s)|^{2} d s, \\
\int_{0}^{t}|\nabla u(s)|^{2} d s=\int_{0}^{t}\left|\int_{0}^{s} \nabla u^{\prime}(\sigma) d \sigma\right|^{2} d s \leq \int_{0}^{t} s \int_{0}^{s}\left|\nabla u^{\prime}(\sigma)\right|^{2} d \sigma d s \\
\leq \int_{0}^{t} \int_{\sigma}^{t} s d s\left|\nabla u^{\prime}(\sigma)\right|^{2} d \sigma \leq \frac{1}{2} \int_{0}^{t}\left(t^{2}-s^{2}\right)\left|\nabla u^{\prime}(\sigma)\right|^{2} d \sigma \\
\leq \frac{1}{2} t^{2} \int_{0}^{t}\left|\nabla u^{\prime}(\sigma)\right|^{2} d \sigma,
\end{gathered}
$$


so that

$$
\int_{0}^{t}\left|G\left(s, \nabla u_{1}(s)\right)-G\left(s, \nabla u_{2}(s)\right)\right|^{2} d s \leq \frac{1}{2}\left\|G_{0}\right\|^{2} t^{2} \int_{0}^{t}\left|\nabla u^{\prime}(\sigma)\right|^{2} d \sigma .
$$

By (3.4), we see

$$
\begin{aligned}
& \left|F\left(s, u_{1}(s), v_{1}^{\prime}(s)\right)-F\left(s, u_{2}(s), v_{2}^{\prime}(s)\right)\right| \\
\leq & \left(c_{1}+1\right) \beta_{1}(s)|\nabla u(s)|+\left(c_{1}+1\right) \beta_{2}(s)\left|\nabla v^{\prime}(s)\right|,
\end{aligned}
$$

so that we can estimate the third term of (4.12) as follows.

$$
\begin{aligned}
& \left|\int_{0}^{t}\left(F\left(s, u_{1}(s), v_{1}^{\prime}(s)\right)-F\left(s, u_{2}(s), v_{2}^{\prime}(s)\right), v^{\prime}(s)\right) d s\right| \\
\leq & \int_{0}^{t}\left|F\left(s, u_{1}(s), v_{1}^{\prime}(s)\right)-F\left(s, u_{2}(s), v_{2}^{\prime}(s)\right)\right|\left|v^{\prime}(s)\right| d s \\
\leq & \int_{0}^{t} \hat{\beta}_{1}(s)|\nabla u(s)|\left|v^{\prime}(s)\right| d s+\int_{0}^{t} \hat{\beta}_{2}(s)\left|\nabla v^{\prime}(s)\right|\left|v^{\prime}(s)\right| d s \\
\leq & \frac{1}{2} \int_{0}^{t}|\nabla u(s)|^{2} d s+\frac{1}{2} \int_{0}^{t} \hat{\beta}_{1}(s)^{2}\left|v^{\prime}(s)\right|^{2} d s \\
& +\frac{\alpha}{4} \int_{0}^{t}\left|\nabla v^{\prime}(s)\right|^{2} d s+\frac{1}{\alpha} \int_{0}^{t} \hat{\beta}_{2}(s)^{2}\left|v^{\prime}(s)\right|^{2} d s . \\
\leq & \frac{1}{2} \int_{0}^{t}|\nabla u(s)|^{2} d s+\frac{\alpha}{4} \int_{0}^{t}\left|\nabla v^{\prime}(s)\right|^{2} d s+\int_{0}^{t} \check{\beta}(s)\left|v^{\prime}(s)\right|^{2} d s,
\end{aligned}
$$

where $\hat{\beta}_{1}(s)=\left(c_{1}+1\right) \beta_{1}(s) \in L^{2}(0, T), \hat{\beta}_{2}(s)=\left(c_{1}+1\right) \beta_{2}(s) \in L^{\infty}(0, T)$ and $\check{\beta}(s)=\frac{1}{2} \hat{\beta}_{1}(s)^{2}+\frac{1}{\alpha} \hat{\beta}_{2}(s)^{2} \in L^{1}(0, T)$. Substituting inequalities (4.13), (4.14) and (4.15) into (4.12) and using (2.3), we can deduce

$$
\begin{aligned}
& \frac{1}{2}\left|v^{\prime}(t)\right|^{2}+\alpha \int_{0}^{t}\left|\nabla v^{\prime}(s)\right|^{2} d s \\
\leq & \frac{1}{2} K_{0} t^{2} \int_{0}^{t}\left|\nabla u^{\prime}(\sigma)\right|^{2} d \sigma+\frac{\alpha}{2} \int_{0}^{t}\left|\nabla v^{\prime}(s)\right|^{2} d s+\frac{1}{2} \int_{0}^{t} B(s)\left|v^{\prime}(s)\right|^{2} d s,
\end{aligned}
$$

where

$$
K_{0}=\frac{1}{\alpha}\left\|G_{0}\right\|^{2}+\frac{1}{2}, \quad B(s)=2 \check{\beta}(s) \in L^{1}(0, T) .
$$

Then from (4.16) it follows that

$$
\left|v^{\prime}(t)\right|^{2} \leq K_{0} t^{2} \int_{0}^{t}\left|\nabla u^{\prime}(\sigma)\right|^{2} d \sigma+\int_{0}^{t} B(s)\left|v^{\prime}(s)\right|^{2} d s .
$$

Now we apply Proposition 2.2 to the inequality (4.17). For the purpose we set

$$
\begin{gathered}
\varphi(t)=\left|v^{\prime}(t)\right|^{2} \in L^{\infty}(0, T), \quad \mu(t)=B(t) \in L^{1}(0, T), \\
\delta(t)=K_{0} t^{2} \int_{0}^{t}\left|\nabla u^{\prime}(\sigma)\right|^{2} d \sigma \in L^{\infty}(0, T) .
\end{gathered}
$$


Since $\delta(t) \geq 0$ is monotonically increasing, Proposition 2.2 implies that

$$
\begin{aligned}
\left|v^{\prime}(t)\right|^{2} & \equiv \varphi(t) \\
& \leq \delta(t)+\delta(t) \int_{0}^{t} \mu(s) \exp \left(\int_{s}^{t} \mu(\sigma) d \sigma\right) d s \\
& =\delta(t)-\delta(t) \int_{0}^{t} \frac{\partial}{\partial s} \exp \left(\int_{s}^{t} \mu(\sigma) d \sigma\right) d s \\
& =\delta(t)-\delta(t)\left(1-\exp \left(\int_{0}^{t} \mu(\sigma) d \sigma\right)\right) \\
& =\delta(t) \exp \left(\int_{0}^{t} \mu(\sigma) d \sigma\right) \\
& \leq K_{0} \exp \left(\|\mu\|_{L^{1}(0, T)}\right) t^{2} \int_{0}^{t}\left|\nabla u^{\prime}(s)\right|^{2} d s \\
& \leq L_{0} t^{2} \int_{0}^{t}\left|\nabla u^{\prime}(s)\right|^{2} d s,
\end{aligned}
$$

where

$$
L_{0}=K_{0} \exp \left(\left(c_{1}+1\right)^{2}\left(\left\|\beta_{1}\right\|^{2}+\frac{2 T}{\alpha}\left\|\beta_{2}\right\|^{2}\right)\right) .
$$

and $\left\|\beta_{1}\right\|=\left\|\beta_{1}\right\|_{L^{2}\left(0, T ; L^{\infty}(\Omega)\right)},\left\|\beta_{2}\right\|=\left\|\beta_{2}\right\|_{L^{\infty}\left(0, T ; L^{\infty}(\Omega)\right)}$. Therefore, (4.16) and (4.18) implies

$$
\begin{aligned}
& \left|v^{\prime}(t)\right|^{2}+\alpha \int_{0}^{t}\left|\nabla v^{\prime}(s)\right|^{2} d s \\
\leq & K_{0} t^{2} \int_{0}^{t}\left|\nabla u^{\prime}(\sigma)\right|^{2} d \sigma+\int_{0}^{t} B(s)\left(L_{0} s^{2} \int_{0}^{s}\left|\nabla u^{\prime}(\sigma)\right|^{2} d \sigma\right) d s \\
\leq & K_{0} t^{2} \int_{0}^{t}\left|\nabla u^{\prime}(\sigma)\right|^{2} d \sigma+L_{0} t^{2} \int_{0}^{t}\left|\nabla u^{\prime}(s)\right|^{2} d s \int_{0}^{T} B(s) d s \\
\leq & M_{0} t^{2} \int_{0}^{t}\left|\nabla u^{\prime}(s)\right|^{2} d s
\end{aligned}
$$

or

$$
\begin{aligned}
& \frac{1}{\alpha}\left|v_{1}^{\prime}(t)-v_{2}^{\prime}(t)\right|^{2}+\int_{0}^{t}\left|\nabla\left(v_{1}^{\prime}(s)-v_{2}^{\prime}(s)\right)\right|^{2} d s \\
\leq & \frac{M_{0}}{\alpha} t^{2} \int_{0}^{t}\left|\nabla\left(u_{1}^{\prime}(s)-u_{2}^{\prime}(s)\right)\right|^{2} d s,
\end{aligned}
$$

where $M_{0}=K_{0}+L_{0}\|B\|_{L^{1}(0, T)}$. Let $t_{0}$ be such that

$$
0<t_{0} \leq T, \quad \sqrt{\frac{M_{0}}{\alpha}} t_{0}<1 .
$$


Starting from some function $u_{1} \in \mathcal{S}$, we define a sequence of functions $\left\{u_{k}\right\}_{k=1}^{\infty}$ inductively by the weak solutions of

$$
\left\{\begin{array}{l}
u_{k+1}^{\prime \prime}+A(t) u_{k+1}^{\prime}=F\left(t, u_{k}, u_{k+1}^{\prime}\right)+\operatorname{div} G\left(t, \nabla u_{k}\right)+f, \quad 0 \leq t \leq T \\
u_{k+1}(0)=y_{0}, u_{k+1}^{\prime}(0)=y_{1} .
\end{array}\right.
$$

Then in view of (4.20)

$$
\begin{aligned}
& \frac{1}{\alpha}\left|u_{k+1}^{\prime}(t)-u_{k}^{\prime}(t)\right|^{2}+\int_{0}^{t}\left|\nabla\left(u_{k+1}^{\prime}(s)-u_{k}^{\prime}(s)\right)\right|^{2} d s \\
\leq & \frac{M_{0}}{\alpha} t_{0}^{2} \int_{0}^{t_{0}}\left|\nabla\left(u_{k}^{\prime}(s)-u_{k-1}^{\prime}(s)\right)\right|^{2} d s, \quad 0 \leq t \leq t_{0},
\end{aligned}
$$

so that

$$
\left\|u_{k+1}^{\prime}-u_{k}^{\prime}\right\|_{L^{2}\left(0, t_{0} ; H_{0}^{1}(\Omega)\right)} \leq \sqrt{\frac{M_{0}}{\alpha}} t_{0}\left\|u_{k}^{\prime}-u_{k-1}^{\prime}\right\|_{L^{2}\left(0, t_{0} ; H_{0}^{1}(\Omega)\right)} .
$$

Hence by $(4.21)$ there exists a function $w \in L^{2}\left(0, t_{0} ; H_{0}^{1}(\Omega)\right)$ such that

$$
u_{k}^{\prime} \rightarrow w \quad \text { in } L^{2}\left(0, t_{0} ; H_{0}^{1}(\Omega)\right) .
$$

Define a function $u$ by

$$
u(t)=y_{0}+\int_{0}^{t} w(s) d s .
$$

Then

$$
\begin{aligned}
\left\|u_{k}(t)-u(t)\right\| & =\left\|\int_{0}^{t} u_{k}^{\prime}(s) d s-\int_{0}^{t} w(s) d s\right\| \\
& \leq \sqrt{t}\left(\int_{0}^{t}\left\|u_{k}^{\prime}(s) d s-w(s)\right\|^{2} d s\right)^{1 / 2} .
\end{aligned}
$$

From (4.22) it easily follows that

$$
\left|u_{k+1}^{\prime}(t)-u_{k}^{\prime}(t)\right| \leq \sqrt{M_{0}} t_{0}\left\|u_{k}^{\prime}-u_{k-1}^{\prime}\right\|_{L^{2}\left(0, t_{0} ; H_{0}^{1}(\Omega)\right)}, \quad 0 \leq t \leq t_{0} .
$$

It follows from (4.23)-(4.26) that

$$
\begin{gathered}
u_{k} \rightarrow u \text { in } C\left(\left[0, t_{0}\right] ; H_{0}^{1}(\Omega)\right), \\
u_{k}^{\prime} \rightarrow u^{\prime} \quad \text { in } L^{2}\left(0, t_{0} ; H_{0}^{1}(\Omega)\right) \cap C\left(\left[0, t_{0}\right] ; L^{2}(\Omega)\right),
\end{gathered}
$$

and also

$$
A(\cdot) u_{k}^{\prime} \rightarrow A(\cdot) u^{\prime} \quad \text { in } \quad L^{2}\left(0, t_{0} ; H^{-1}(\Omega)\right) .
$$

Since

$$
\begin{aligned}
& \left\|G\left(t, \nabla u_{k}(t, \cdot)\right)-G(t, \nabla u(t, \cdot))\right\|^{2} \\
\leq & \left\|G_{0}\right\|^{2}\left\|u_{k}(t)-u(t)\right\|_{H_{0}^{1}(\Omega)}^{2} \rightarrow 0 \quad \text { uniformly in } t \in\left[0, t_{0}\right]
\end{aligned}
$$

by (4.6), we obtain

$$
\operatorname{div} G\left(\cdot, \nabla u_{k}\right)-\operatorname{div} G(\cdot, \nabla u) \rightarrow 0 \quad \text { in } \quad C\left(\left[0, t_{0}\right] ; H^{-1}(\Omega)\right) .
$$


By (3.4), we have

$$
\begin{aligned}
& \left|F\left(t, u_{k}(t, \cdot), u_{k+1}^{\prime}(t, \cdot)\right)-F\left(t, u(t, \cdot), u^{\prime}(t, \cdot)\right)\right|^{2} \\
\leq & 2\left(c_{1}+1\right)^{2}\left(\beta_{1}(t)^{2}\left|\nabla u_{k}(t)-\nabla u(t)\right|^{2}+\beta_{2}(t)^{2}\left|\nabla u_{k+1}^{\prime}(t)-\nabla u^{\prime}(t)\right|^{2}\right),
\end{aligned}
$$

so that

$$
\begin{aligned}
& \int_{0}^{t_{0}}\left|F\left(t, u_{k}(t, \cdot), u_{k+1}^{\prime}(t, \cdot)\right)-F\left(t, u(t, \cdot), u^{\prime}(t, \cdot)\right)\right|^{2} d t \\
\leq & 2\left(c_{1}+1\right)^{2}\left(\int_{0}^{t_{0}} \beta_{1}(t)^{2} d t\right)\left\|u_{k}-u\right\|_{C\left([0, T] ; H_{0}^{1}(\Omega)\right)}^{2} \\
& +2\left(c_{1}+1\right)^{2}\left\|\beta_{2}\right\|^{2}\left\|u_{k+1}^{\prime}(t)-u^{\prime}(t)\right\|_{L^{2}\left(0, T ; H_{0}^{1}(\Omega)\right)}^{2} .
\end{aligned}
$$

This proves that

$$
F\left(\cdot, u_{k}, u_{k+1}^{\prime}\right)-F\left(\cdot, u, u^{\prime}\right) \rightarrow 0 \quad \text { in } \quad L^{2}\left(0, t_{0} ; L^{2}(\Omega)\right) .
$$

By virtue of (4.29), (4.30) and (4.32) one observes

$$
\begin{aligned}
u_{k+1}^{\prime \prime} & =F\left(\cdot, u_{k}, u_{k+1}^{\prime}\right)+\operatorname{div} G\left(\cdot, \nabla u_{k}\right)-A(\cdot) u_{k+1}^{\prime}+f \\
& \rightarrow F\left(\cdot, u, u^{\prime}\right)+\operatorname{div} G(\cdot, \nabla u)-A(\cdot) u^{\prime}+f \quad \text { in } L^{2}\left(0, t_{0} ; H^{-1}(\Omega)\right) .
\end{aligned}
$$

Hence

$$
u^{\prime \prime} \in L^{2}\left(0, t_{0} ; H^{-1}(\Omega)\right), \quad u_{k}^{\prime \prime} \rightarrow u^{\prime \prime} \quad \text { in } \quad L^{2}\left(0, t_{0} ; H^{-1}(\Omega)\right) .
$$

Therefore

$$
u^{\prime \prime}-\operatorname{div} G(\cdot, \nabla u)+A(\cdot) u^{\prime}=F\left(\cdot, u, u^{\prime}\right)+f \quad \text { in } \quad L^{2}\left(0, t_{0} ; H^{-1}(\Omega)\right) .
$$

In view of (4.27) and (4.28) it is obvious that

$$
u(0)=y_{0}, \quad u^{\prime}(0)=y_{1} .
$$

By repeating the standard argument in Showalter [11], we can verify that this $u$ is a weak solution of $(4.1)$ in the interval $\left[0, t_{0}\right]$.

To continue this solution, consider the mapping which maps $u \in W^{1,2}\left(t_{0}, T\right.$; $\left.H_{0}^{1}(\Omega)\right)$ satisfying $u\left(t_{0}\right)=y\left(t_{0}\right), u^{\prime}\left(t_{0}\right)=y^{\prime}\left(t_{0}\right)$ to the weak solution $v$ of the problem

$$
\left\{\begin{array}{l}
v^{\prime \prime}+A(t) v^{\prime}=F\left(t, u, v^{\prime}\right)+\operatorname{div} G(t, \nabla u)+f, \quad t_{0}<t \leq T \\
v\left(t_{0}\right)=y\left(t_{0}\right), v^{\prime}\left(t_{0}\right)=y^{\prime}\left(t_{0}\right),
\end{array}\right.
$$

where $y$ is the weak solution of (3.1) which is already obtained in $\left[0, t_{0}\right]$,

Let $u_{i} \in W^{1,2}\left(t_{0}, T ; H_{0}^{1}(\Omega)\right), u_{i}\left(t_{0}\right)=y\left(t_{0}\right), u_{i}^{\prime}\left(t_{0}\right)=y^{\prime}\left(t_{0}\right), i=1,2$, and $v_{i}, i=1,2$, be the solution of (4.33) with $u$ replaced by $u_{i}$. Then we can show analogously to $(4.20)$

$$
\begin{aligned}
& \frac{1}{\alpha}\left|v_{1}^{\prime}(t)-v_{2}^{\prime}(t)\right|^{2}+\int_{t_{0}}^{t}\left|\nabla\left(v_{1}^{\prime}(s)-v_{2}^{\prime}(s)\right)\right|^{2} d s \\
\leq & \frac{M_{0}}{\alpha}\left(t-t_{0}\right)^{2} \int_{t_{0}}^{t}\left|\nabla\left(u_{1}^{\prime}(s)-u_{2}^{\prime}(s)\right)\right|^{2} d s .
\end{aligned}
$$


Consequently we can continue $y$ to the interval $\left[t_{0}, 2 t_{0}\right]$. Repeating this process a global solution is obtained.

Lastly in the proof, we shall show the energy inequality (3.8). By the energy equality (4.3), the weak solution $y$ of (3.1) satisfies

$$
\begin{aligned}
& \frac{1}{2}\left|y^{\prime}(t)\right|^{2}+\int_{0}^{t} a\left(s ; y^{\prime}(s), y^{\prime}(s)\right) d s \\
= & \frac{1}{2}\left|y_{1}\right|^{2}+\int_{0}^{t}\left(F\left(s, y(s), y^{\prime}(s)\right), y^{\prime}(s)\right) d s+\int_{0}^{t}\left\langle f(s), y^{\prime}(s)\right\rangle d s \\
& +\int_{0}^{t}\left\langle\operatorname{div} G(s, \nabla y(s)), y^{\prime}(s)\right\rangle d s, \quad \forall t \in[0, T] .
\end{aligned}
$$

By repeating similar calculations for (4.13) (4.14), we have

$$
\begin{aligned}
& \left|\int_{0}^{t}\left(G(s, \nabla y(s)), \nabla y^{\prime}(s)\right) d s\right| \\
\leq & \frac{1}{\alpha}\left\|G_{0}\right\|^{2} \int_{0}^{t}|\nabla y(s)|^{2} d s+\frac{\alpha}{4} \int_{0}^{t}\left|\nabla y^{\prime}(s)\right|^{2} d s .
\end{aligned}
$$

Also, by (3.4) and (3.5), we use Schwarz inequality to have

$$
\begin{aligned}
& \left|\int_{0}^{t}\left(F\left(s, y(s), y^{\prime}(s)\right), y^{\prime}(s)\right) d s\right| \\
\leq & \int_{0}^{t}\left|F\left(s, y(s), y^{\prime}(s)\right)\right|\left|y^{\prime}(s)\right| d s \\
\leq & \int_{0}^{t}\left(\left|F\left(s, y(s), y^{\prime}(s)\right)-F(s, y(s), 0)\right|+|F(s, y(s), 0)|\right)\left|y^{\prime}(s)\right| d s \\
\leq & \int_{0}^{t} \hat{\beta}_{2}(s)\left|\nabla y^{\prime}(s)\right|\left|y^{\prime}(s)\right| d s+\int_{0}^{t} \hat{\gamma}(s)|\nabla y(s)|\left|y^{\prime}(s)\right| d s \\
\leq & \frac{\alpha}{8} \int_{0}^{t}\left|\nabla y^{\prime}(s)\right|^{2} d s+\int_{0}^{t} \check{\gamma}(s)\left|y^{\prime}(s)\right|^{2} d s+\frac{1}{2} \int_{0}^{t}|\nabla y(s)|^{2} d s,
\end{aligned}
$$

where $\hat{\beta}_{2}(s)=\left(c_{1}+1\right) \beta_{2}(s) \in L^{\infty}(0, T), \hat{\gamma}(s)=\left(c_{1}+1\right) \gamma(s) \in L^{2}(0, T)$ and $\check{\gamma}(s)=\frac{1}{2} \hat{\gamma}(s)^{2}+\frac{2}{\alpha} \hat{\beta}_{2}(s)^{2} \in L^{1}(0, T)$. It is easy to see that

$$
\begin{aligned}
\left|\int_{0}^{t}\left\langle f(s), y^{\prime}(s)\right\rangle d s\right| & \leq \int_{0}^{t}\|f(s)\|_{H^{-1}(\Omega)}\left\|y^{\prime}(s)\right\| d s \\
& \leq \frac{2}{\alpha} \int_{0}^{t}\|f(s)\|_{H^{-1}(\Omega)}^{2} d s+\frac{\alpha}{8} \int_{0}^{t}\left|\nabla y^{\prime}(s)\right|^{2} d s .
\end{aligned}
$$

Hence, from the equality (4.34) together with the inequalities (4.35)-(4.37) it follows that

$$
\left|y^{\prime}(t)\right|^{2}+\alpha \int_{0}^{t}\left|\nabla y^{\prime}(s)\right|^{2} d s
$$




$$
\begin{aligned}
\leq & \left|y_{1}\right|^{2}+M_{0} \int_{0}^{t}|\nabla y(s)|^{2} d s+\int_{0}^{t} \Gamma(s)\left|y^{\prime}(s)\right|^{2} d s \\
& +\frac{4}{\alpha} \int_{0}^{t}\|f(s)\|_{H^{-1}(\Omega)}^{2} d s
\end{aligned}
$$

where

$$
M_{0}=2\left(\frac{1}{\alpha}\left\|G_{0}\right\|^{2}+\frac{1}{2}\right), \quad \Gamma(s)=2 \check{\gamma}(s) \in L^{1}(0, T) .
$$

Since $y(t)=y_{0}+\int_{0}^{t} y^{\prime}(s) d s$, we obtain

(4.39) $|\nabla y(t)|^{2} \leq 2\left(\left|\nabla y_{0}\right|^{2}+\left|\int_{0}^{t} \nabla y^{\prime}(s) d s\right|^{2}\right) \leq 2\left(\left\|y_{0}\right\|^{2}+t \int_{0}^{t}\left|\nabla y^{\prime}(s)\right|^{2} d s\right)$,

so that

$$
\begin{aligned}
\int_{0}^{t}|\nabla y(s)|^{2} d s & \leq 2 t\left\|y_{0}\right\|^{2}+2 \int_{0}^{t} s \int_{0}^{s}\left|\nabla y^{\prime}(\sigma)\right|^{2} d \sigma d s \\
& \leq 2 t\left\|y_{0}\right\|^{2}+\int_{0}^{t}\left(t^{2}-\sigma^{2}\right)\left|\nabla y^{\prime}(\sigma)\right|^{2} d \sigma .
\end{aligned}
$$

Then by (4.38) and (4.40) we arrive at the inequality

$$
\left|y^{\prime}(t)\right|^{2}+\alpha \int_{0}^{t}\left|\nabla y^{\prime}(s)\right|^{2} d s \leq \tilde{\delta}(t)+M_{0} \int_{0}^{t}\left(t^{2}-\sigma^{2}\right)\left|\nabla y^{\prime}(\sigma)\right|^{2} d \sigma,
$$

where

$$
\tilde{\delta}(t)=\left|y_{1}\right|^{2}+\frac{4}{\alpha} \int_{0}^{t}\|f(s)\|_{H^{-1}(\Omega)}^{2} d s+2 M_{0}\left\|y_{0}\right\|^{2} t+\int_{0}^{t} \Gamma(s)\left|y^{\prime}(s)\right|^{2} d s .
$$

From (4.41) it follows that

$$
\int_{0}^{t}\left|\nabla y^{\prime}(s)\right|^{2} d s-\frac{M_{0}}{\alpha} \int_{0}^{t}\left(t^{2}-\sigma^{2}\right)\left|\nabla y^{\prime}(\sigma)\right|^{2} d \sigma \leq \frac{1}{\alpha} \tilde{\delta}(t) .
$$

Hence by (4.42),

$$
\begin{aligned}
& \frac{d}{d t}\left\{e^{-M_{0} t^{2} / \alpha} \int_{0}^{t}\left(t^{2}-\sigma^{2}\right)\left|\nabla y^{\prime}(\sigma)\right|^{2} d \sigma\right\} \\
= & 2 t e^{-M_{0} t^{2} / \alpha}\left\{\int_{0}^{t}\left|\nabla y^{\prime}(\sigma)\right|^{2} d \sigma-\frac{M_{0}}{\alpha} \int_{0}^{t}\left(t^{2}-\sigma^{2}\right)\left|\nabla y^{\prime}(\sigma)\right|^{2} d \sigma\right\} \\
\leq & \frac{2 t}{\alpha} e^{-M_{0} t^{2} / \alpha} \tilde{\delta}(t) .
\end{aligned}
$$

Integration of both sides of (4.43) from 0 to $t$ yields

$$
\begin{aligned}
& e^{-M_{0} t^{2} / \alpha} \int_{0}^{t}\left(t^{2}-\sigma^{2}\right)\left|\nabla y^{\prime}(\sigma)\right|^{2} d \sigma \leq \frac{2}{\alpha} \int_{0}^{t} \tau e^{-M_{0} \tau^{2} / \alpha} \tilde{\delta}(\tau) d \tau \\
& \leq \frac{2}{\alpha}\left(\int_{0}^{t} \tau e^{-M_{0} \tau^{2} / \alpha} d \tau\right) \tilde{\delta}(t)=\frac{1}{M_{0}}\left(1-e^{-M_{0} t^{2} / \alpha}\right) \tilde{\delta}(t),
\end{aligned}
$$


where we note that $\tilde{\delta}(\tau) \leq \tilde{\delta}(t), \forall \tau \in[0, t]$. The inequality (4.44) implies

$$
\int_{0}^{t}\left(t^{2}-\sigma^{2}\right)\left|\nabla y^{\prime}(\sigma)\right|^{2} d \sigma \leq \frac{1}{M_{0}}\left(e^{M_{0} t^{2} / \alpha}-1\right) \tilde{\delta}(t) \leq \frac{M_{1}}{M_{0}} \tilde{\delta}(t),
$$

where $M_{1}=e^{M_{0} T^{2} / \alpha}$. From (4.41) and (4.45) it follows that

$$
\left|y^{\prime}(t)\right|^{2}+\alpha \int_{0}^{t}\left|\nabla y^{\prime}(s)\right|^{2} d s \leq\left(1+M_{1}\right) \tilde{\delta}(t),
$$

or exactly

$$
\begin{aligned}
& \left|y^{\prime}(t)\right|^{2}+\alpha \int_{0}^{t}\left|\nabla y^{\prime}(s)\right|^{2} d s \\
\leq & M_{2}\left(\left|y_{1}\right|^{2}+\frac{4}{\alpha} \int_{0}^{t}\|f(s)\|_{H^{-1}(\Omega)}^{2} d s+2 M_{0}\left\|y_{0}\right\|^{2} t\right) \\
& +\int_{0}^{t} M_{2} \Gamma(s)\left|y^{\prime}(s)\right|^{2} d s,
\end{aligned}
$$

where $M_{2}=1+M_{1}$. In order to obtain the desired energy inequality, we set

$$
\begin{gathered}
\varphi(t)=\left|y^{\prime}(t)\right|^{2} \in L^{\infty}(0, T), \quad \mu(t)=M_{2} \Gamma(t) \in L^{1}(0, T), \\
\delta(t)=M_{2}\left(\left|y_{1}\right|^{2}+\frac{4}{\alpha} \int_{0}^{t}\|f(s)\|_{H^{-1}(\Omega)}^{2} d s+2 M_{0}\left\|y_{0}\right\|^{2} t\right) \in L^{\infty}(0, T) .
\end{gathered}
$$

Then the inequality (4.47) implies

$$
\varphi(t) \leq \int_{0}^{t} \mu(\sigma) \varphi(\sigma) d \sigma+\delta(t), \quad \forall t \in[0, T] .
$$

Since $\delta(t)$ is monotonically increasing, by Proposition 2.2 we obtain that

$$
\begin{aligned}
& \varphi(t) \equiv\left|y^{\prime}(t)\right|^{2} \\
\leq & \delta(t)+\delta(t) \int_{0}^{t} \mu(s) \exp \left(\int_{s}^{t} \mu(\sigma) d \sigma\right) d s=\delta(t) \exp \left(\int_{0}^{t} \mu(s) d s\right) \\
\leq & \delta(t) \exp \left(\left\|M_{2} \Gamma\right\|_{L^{1}(0, T)}\right) \equiv M_{3} \delta(t) .
\end{aligned}
$$

Therefore, by (4.48) the inequality (4.47) implies

$$
\begin{gathered}
\left|y^{\prime}(t)\right|^{2}+\alpha \int_{0}^{t}\left|\nabla y^{\prime}(s)\right|^{2} d s \leq \delta(t)+\int_{0}^{t} M_{2} M_{3} \Gamma(s) \delta(s) d s \\
\leq \delta(t)+M_{2} M_{3}\left(\int_{0}^{T} \Gamma(s) d s\right) \delta(t) \leq\left(1+M_{2} M_{3}\|\Gamma\|_{L^{1}(0, T)}\right) \delta(t) .
\end{gathered}
$$

From (4.39), it is easily seen that

$$
|\nabla y(t)|^{2} \leq 2\left(\left\|y_{0}\right\|^{2}+T \int_{0}^{t}\left|\nabla y^{\prime}(s)\right|^{2} d s\right),
$$


Combining (4.49) and (4.50), we can verify that

$$
\left|y^{\prime}(t)\right|^{2}+|\nabla y(t)|^{2}+\int_{0}^{t}\left|\nabla y^{\prime}(s)\right|^{2} d s \leq 2\left\|y_{0}\right\|^{2}+C \delta(t) \leq C\left(\left\|y_{0}\right\|^{2}+\delta(T)\right)
$$

for some $C>2$ depending only on $\alpha>0, G_{0}, \beta_{1}, \beta_{2}, \gamma$ and $T>0$. This proves the energy inequality (3.8).

Proof of Theorem 3.2. Let $y\left(p_{1}\right)$ and $y\left(p_{2}\right)$ be the weak solutions of (4.1) corresponding to $p_{1} \in P$ and $p_{2} \in P$, respectively. Set $\varphi=y\left(p_{1}\right)-y\left(p_{2}\right)$. Then $\varphi$ satisfies

$$
\left\{\begin{array}{l}
\varphi^{\prime \prime}(t)-\operatorname{div}\left(G\left(t, \nabla y\left(p_{1}\right)\right)-G\left(t, \nabla y\left(p_{2}\right)\right)\right)+A(t) \varphi^{\prime}(t) \\
\quad-\left(F\left(t, y\left(p_{1}\right), y^{\prime}\left(p_{1}\right)\right)-F\left(t, y\left(p_{2}\right), y^{\prime}\left(p_{2}\right)\right)\right)=f_{1}-f_{2}, \quad 0 \leq t \leq T \\
\varphi(0)=y_{0}^{1}-y_{0}^{2}, \quad \varphi^{\prime}(0)=y_{1}^{1}-y_{1}^{2}
\end{array}\right.
$$

Since $G$ and $F$ satisfy

$$
\left|G\left(t, \nabla y\left(p_{1}\right)\right)-G\left(t, \nabla y\left(p_{2}\right)\right)\right| \leq \| G_{0}||\left|\nabla y\left(p_{1}\right)-\nabla y\left(p_{2}\right)\right|
$$

and

$$
\begin{aligned}
& \left|F\left(t, y\left(p_{1}\right), y^{\prime}\left(p_{1}\right)\right)-F\left(t, y\left(p_{2}\right), y^{\prime}\left(p_{2}\right)\right)\right| \\
\leq & \left(c_{1}+1\right)\left(\beta_{1}(t)\left(\left|\nabla y\left(p_{1}\right)-\nabla y\left(p_{2}\right)\right|+\beta_{2}(t)\left|\nabla y^{\prime}\left(p_{1}\right)-\nabla y^{\prime}\left(p_{2}\right)\right|\right)\right.
\end{aligned}
$$

for a.e. $t \in[0, T]$, we can repeat the same calculations as in the proof of Theorem 3.1 to have the following estimate

$$
\begin{aligned}
& |\nabla \varphi(t)|^{2}+\left|\varphi^{\prime}(t)\right|^{2}+\int_{0}^{t}\left|\nabla \varphi^{\prime}\right|^{2} d s \\
\leq & C\left(\left\|y_{0}^{1}-y_{0}^{2}\right\|^{2}+\left|y_{1}^{1}-y_{1}^{2}\right|^{2}+\left\|f_{1}-f_{2}\right\|_{L^{2}\left(0, T ; H^{-1}(\Omega)\right)}^{2} .\right.
\end{aligned}
$$

This proves the strong Lipshitz continuity of solution mapping $p=\left(y_{0}, y_{1}, f\right) \rightarrow$ $y(p)$ of $P$ into $W(0, T)$.

At the end of this paper, we shall give one application of Theorem 3.1 to the variational problem. We consider the original equation (1.1) of motion of membrane with strong viscosity. It is easily verified that $G(t, \nabla y)=G(\nabla y)=$ $\frac{\nabla y}{\sqrt{1+|\nabla y|^{2}}}$ and $-\mu \Delta y=-\operatorname{div}(\mu \nabla y)=A(t) y$ satisfy (G-i)-(G-iii) and (2.3) with $\alpha=\mu$, respectively. Then for each $p=\left(y_{0}, y_{1}, f\right) \in P$ there exists a unique solution $y(p)$ of (1.1). It is suggested in Hwang and Nakagiri [8] that $y(p)$ is Gâteaux differentiable in $p$ and the derivative $z=\partial y(p) q, q=\left(z_{0}, z_{1}, g\right) \in P$ 
is characterized by the variational equation

$$
\begin{cases}\frac{\partial^{2} z}{\partial t^{2}}-\operatorname{div}\left(\frac{\nabla z}{\sqrt{1+|\nabla y(p)|^{2}}}-\nabla y(p) \frac{\nabla y(p) \cdot \nabla z}{\left(1+|\nabla y(p)|^{2}\right)^{3 / 2}}\right) \\ z=0 \quad \text { on } \Sigma, & -\mu \Delta \frac{\partial z}{\partial t}=g, \quad \text { in } Q \\ z(0, x)=z_{0}(x), \quad \frac{\partial z}{\partial t}(0, x)=z_{1}(x) & \text { in } \Omega .\end{cases}
$$

Really, this $z$ should be the Gâteaux derivative of $y(p)$ at $p$ in the direction $q=\left(z_{0}, z_{1}, g\right) \in P$. The existence and uniqueness of (4.53) can be shown as an easy application of Theorem 3.1, since

$$
G(t, x, \nabla z)=\frac{\nabla z}{\sqrt{1+|\nabla y(p ; t, x)|^{2}}}-\nabla y(p ; t, x) \frac{\nabla y(p ; t, x) \cdot \nabla z}{\left(1+|\nabla y(p ; t, x)|^{2}\right)^{3 / 2}}
$$

satisfies the inequality

$$
|G(t, \nabla z)| \leq 2|\nabla z|, \quad \forall t \in[0, T], \forall z \in H_{0}^{1}(\Omega) .
$$

\section{References}

[1] H. T. Banks, R. C. Smith, and Y. Wang, Smart Material Structures, Modeling, Estimation and Control, RAM, John Wiley and Sons, Masson, 1996.

[2] R. Dautray and J. L. Lions, Mathematical Analysis and Numerical Methods for Science and Technology, Vol. 5, Evolution Problems I, Springer-Verlag, 1992.

[3] D. Gilbarg and N. S. Trudinger, Elliptic Partial Differential Equations of Second Order, Springer-Verlag, Berlin-Heidelberg-New York, 1977.

[4] J. Greenberg, On the existence, uniqueness, and stability of solutions of the equation $\rho_{0} X_{t t}=E\left(X_{x}\right) X_{x x}+\lambda X_{x x t}$, J. Math. Anal. Appl. 25 (1969), 575-591.

[5] J. Greenberg, R. MacCamy, and V. Mizel, On the existence, uniqueness, and stability of solutions of the equation $\sigma^{\prime}\left(u_{x}\right) u_{x x}+\lambda u_{x t x}=\rho_{0} u_{t t}$, J. Math. Mech. $17(1967 / 1968)$, 707-728.

[6] J.-H., Ha, S. Nakagiri, and H. Tanabe, Fréchet differentiability of solution mappings for semilinear second order evolution equations, J. Math. Anal. Appl. 346 (2008), no. 2, $374-383$.

[7] J. S. Hwang and S. Nakagiri, Weak solutions of the equation of motion of membrane with strong viscosity, J. Korean Math. Soc. 44 (2007), no. 2, 443-453.

[8] _ Optimal control problems for the equation of motion of membrane with strong viscosity, J. Math. Anal. Appl. 321 (2006), no. 1, 327-342.

[9] _ Parameter identification problems for the equation of motion of membrane with strong viscosity, J. Math. Anal. Appl. 342 (2008), no. 1, 125-134.

[10] T. Kobayashi, H. Pecher, and Y. Shibata, On a global in time existence theorem of smooth solutions to a nonlinear wave equation with viscosity, Math. Ann. 296 (1993), no. 2, 215-234.

[11] R. E. Showalter, Hilbert Space Method for Partial Differential Equations, Pitman, London, 1977.

[12] R. Temam, Infinite-Dimensional Dynamical Systems in Mechanics and Physis, Second Edition, Applied Mathematical Sciences. Vol. 68, Springer-Verlag, Berlin-HeidelbergNew York, 1997. 
[13] Q.-F. Wang and S. Nakagiri, Weak solutions of nonlinear parabolic evolution problems with uniform Lipschitz continuous nonlinearities, Mem. Grad. School Sci. \& Technol., Kobe Univ. 19-A (2001), 83-96.

\section{JIN-SOO HWANG}

Department of Mathematics Education

College of Education

DAEgu UnIVERSITY

Gyeongbuk 712-714, KoreA

E-mail address: jshwang@daegu.ac.kr

SHIN-ICHI NAKAGIRI

Department of Applied Mathematics

FACUlty OF EngineERING

KoBe UNIVERSITY

KOBE 657-8501, JAPAN

E-mail address: nakagiri@cs.kobe-u.ac.jp

HiROKI TANABE

EMERITUs Professor of Osaka University

Hiraisanso 12-13, TAKARAZUKa 665-0817, JAPAN

E-mail address: h7tanabe@jttk.zak.ne.jp 